\title{
The Roles of circRNAs in Intervertebral Disc Degeneration: Inflammation, Extracellular Matrix Metabolism, and Apoptosis
}

\author{
Hao Li, ${ }^{1,2}$ Lijun Tian, ${ }^{2,3}$ Jianhua Li, ${ }^{4}$ Yongjin Li ${ }^{1,2}$ Lilong Du, ${ }^{1,2}$ Zhenxin Huo, ${ }^{1,2}$ \\ and Baoshan $\mathrm{Xu}$ iD ${ }^{1,2}$ \\ ${ }^{1}$ Department of Minimally Invasive Spine Surgery, Tianjin Hospital, No. 406, Jiefangnan Road, Hexi District, Tianjin, China 300211 \\ ${ }^{2}$ Graduate School, Tianjin Medical University, 22 Qixiangtai Road, Tianjin, China \\ ${ }^{3}$ Department of Orthopaedics, The First Affiliated Hospital of Baotou Medical College, Inner Mongolia University of Science \\ and Technology, Baotou, Inner Mongolia, China 014010 \\ ${ }^{4}$ Department of Orthopaedics, Tianjin Haihe Hospital, Tianjin 300350, China
}

Correspondence should be addressed to Baoshan Xu; baoshanxu99@tmu.edu.cn

Received 23 November 2021; Accepted 19 January 2022; Published 11 February 2022

Academic Editor: Maria Beatrice Morelli

Copyright (c) 2022 Hao Li et al. This is an open access article distributed under the Creative Commons Attribution License, which permits unrestricted use, distribution, and reproduction in any medium, provided the original work is properly cited.

\begin{abstract}
Low back pain (LBP) is seriously harmful to human health and produces heavy economic burden. And most scholars hold that intervertebral disc degeneration (IDD) is the primary cause of LBP. With the study of IDD, aberrant expression of gene has become an important pathogenic factor of IDD. Circular RNAs (circRNAs), as a kind of noncoding RNA (ncRNA), participate in the regulation of genetic transcription and translation and further affect the expression of inflammatory cytokine, metabolism of extracellular matrix (ECM), the proliferation and apoptosis of cells, etc. Therefore, maybe it will become a new therapeutic target for IDD. At present, our understanding of the mechanism of circRNAs in IDD is limited. The purpose of this review is to summarize the mechanism and related signaling pathways of circRNAs in IDD reported in the past. Particularly, the roles of circRNAs in inflammation, ECM metabolism, and apoptosis are emphasized.
\end{abstract}

\section{Introduction}

Low back pain (LBP) is one of the most common symptoms of orthopedic patients all over the world and causes a heavy burden [1-3]. According to the Global Burden of Diseases, Injuries, and Risk Factors Study 2017 (GBD 2017), low back pain was regarded as the primary cause of years lived with disability (YLDs) counts in 2017 [4]. Besides, a systematic analysis for the GBD 2019 indicated that low back pain had the highest need of rehabilitation services in 134 of the 204 countries and brought serious economic burden [5]. From the etiology of LBP, intervertebral disc degeneration (IDD) is mainly responsible for the LBP and is a key target for the diagnosis and therapy of LBP [6-8]. It is well known that aging, trauma, and genetic predisposition may change the function and structure of the nucleus pulposus cells (NPCs), thus disc degeneration and pain $[9,10]$. Previously, the chief suspected risk factor for intervertebral disc degeneration was heavy physical loading, which was commonly viewed as a wear-and-tear phenomenon [11]. However, at present, more researches suggested that IDD is genetically driven [11-14]. They found that there are many significantly differentially expressed genes (DEGs) in IDD tissues compared with normal tissues [15-18]. Among this, DEGs, noncoding RNAs (ncRNAs), as an important regulatory element, play critical roles in IDD [19]. Therefore, we suppose that these ncRNAs will provide a new direction for diagnosis and treatment of IDD. More recently, the reports of circular RNAs (circRNAs) add fuel to the ncRNA research and indicate that circRNAs can regulate pathological process of IDD as competing endogenous RNAs (ceRNAs), which include inflammation, ECM metabolism and NPC proliferation, autograph, and apoptosis [20-22]. However, at present, the mechanism of 
circRNAs is not completely clear. The researches of circRNAs have great potential and may become a new target for the treatment of IDD.

\section{The Mechanism of Intervertebral Disc Degeneration}

The central nucleus pulposus (NP), peripheric annulus fibrosus (AF), and cartilaginous end plates (CEP) constitute the intervertebral disc (IVD), which maintain the structure and function of the spine [23].

The IVD includes multiple collagen types, chief among them are type I and type II collagen [24], and there is a gradual transition from type II to type I from the central nucleus pulposus to the peripheral annulus fibrosus [25]. The disc possesses a variety of proteoglycans (PGs) in its extracellular matrix (ECM), and aggrecan is the most abundant proteoglycan in the disc, which is responsible for maintaining the moisture and mechanical load of IVD and is precondition for IVD to exert its physiological function and absorb stress [24, 26-28]. Proteoglycans can make the NP highly hydrated with high osmolarity, enabling the IVD to buffer pressure loads and to maintain morphology [29-31]. Early in the disc degeneration, the nucleus pulposus loses proteoglycans, leading to the decline of osmotic pressure in IVD [32]. This affects its biomechanical function [30]. The correlation study suggested that the proteoglycan content positively correlated with the viscoelastic properties of the disc; however, there was no correlation with the collagen content. These results suggest that the proteoglycan play a dominant role for maintaining the properties of IVD [29]. The AF is composed of multiple concentric annulus, resisting the stress in all directions [33,34]. The cartilage endplate is a hyaline cartilage located on the upper and lower part of the intervertebral disc [35]. It is important that the nutrients of the NP cells mainly come from the microvascular system in the cartilage endplate, which passes through the endplate and then spreads through the nucleus pulposus matrix [36, 37]. However, there is almost no vascular supply in the adult disc. The intervertebral disc is also regarded as the largest avascular tissue in adults $[38,39]$.

Currently, people's understanding of IDD is limited. However, there are abundant evidences indicated that various factors, such as genetic factors, mechanical stress, trauma, fatty, and smoking, are associated with the pathomechanism of IDD [31]. Finally, the above factors disturb homeostasis, changing the morphology and function of the IVD and ultimately resulting in herniation and pain [40]. During the process, degradation of the ECM, inflammation, and apoptosis play a dominant role [41] (Figure 1). Significantly, circRNAs, as a kind of noncoding RNAs, participate in the occurrence and development of IDD [19, 21, $42-44]$. The purpose of this review is to summarize the types and functions of circRNAs involved in the pathological process of intervertebral disc. We also speculated that circRNAs may become a new target for the diagnosis or treatment of IDD in the future $[45,46]$.

\section{The Characteristic of circRNAs}

ncRNAs are important regulatory elements and play critical roles in diverse diseases [19, 47, 48], which include microRNAs (miRNAs), long ncRNAs (lncRNAs), and the recently discovered circRNAs $[49,50]$. Sanger et al. first discovered that circRNAs are a kind of ncRNAs with high thermal stability in plant-infected virions in 1976 [51]. circRNAs have no free $3^{\prime}$ or $5^{\prime}$ end, which forms a closed loop structure with $5^{\prime}$ and $3^{\prime}$ ends joining together [52, 53]. Currently, in terms of the type and quantity of the parental gene, many scholars have identified seven types of circRNAs, and exonic circRNAs are the most common type of circRNAs [51]. With increasing researches, circRNAs are considered to have several possible functions, including binding to mRNA competitively and regulating genetic transcription and translation [20, 22, 42, 52, 54]. With the deepening of the study of circRNAs, the roles of circRNAs in the occurrence and development of the disease have been gradually recognized [55-57]. It could even serve as a marker for disease diagnosis [58-60]. In the meantime, increasing evidences show that circRNAs are also closely associated with the process of IDD. Zhang et al. [19] investigated a series of public datasets (GSE67566, GSE56081, and GSE63492) and identified 586 circular RNAs that were expressed differently in IDD compared with normal discs. Gene Ontology (GO) analysis demonstrated that these differently expressed circRNAs were involved in the regulation of cellular component, gene expression, and metabolic processes. Wang et al. [61] also identified 7294 circRNAs significantly differently expressed in degenerated human NPCs by microarray analysis. Besides, with the innovation of technology, the function of circRNAs has been gradually verified in the cellular and molecular level.

\section{The Roles of circRNAs in Intervertebral Disc Degeneration}

4.1. Inflammation. The increase in levels of the inflammatory cytokines is the character of IDD [62]. Numerous studies also have revealed higher expression of the proinflammatory cytokines TNF- $\alpha$, IL- $1 \alpha$, IL- $1 \beta$, and IL- 6 in degenerative disc $[6,63-69]$. These cytokines promote ECM degradation, chemokine production, and change of the phenotype of cells [70-72], finally leading to the degeneration of IVD, as well as disc herniation and radicular pain [6].

Recently, increasing evidences indicated that circRNAs are correlation with the production of inflammatory cytokines. Song et al. [73] studies have shown that circRNA_0000253 can be used as ceRNA to combine with miRNA-141-5p, thus promoting the synthesis and secretion of IL- $1 \beta$ which stimulate oxidative stress response and expression of apoptotic proteins such as caspase $3 / 7 / 9$ and promote the expression of matrix proteases (MMP-3 and ADAMTS5), while inhibit the synthesis of COL-II and aggrecan. In addition, Guo et al. [44] found that the competitive binding of circRNA FAM169A and miRNA583 promoted the expression of BTRC, thus promoting the secretion of inflammatory factors. On the contrary, Heng 


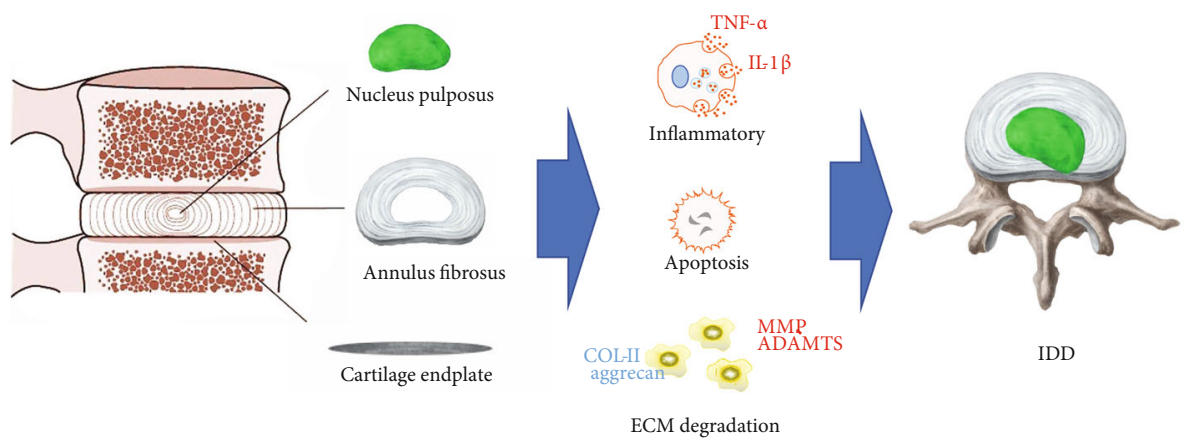

FIGURE 1: The component of intervertebral disc and the major pathologic process of degeneration.

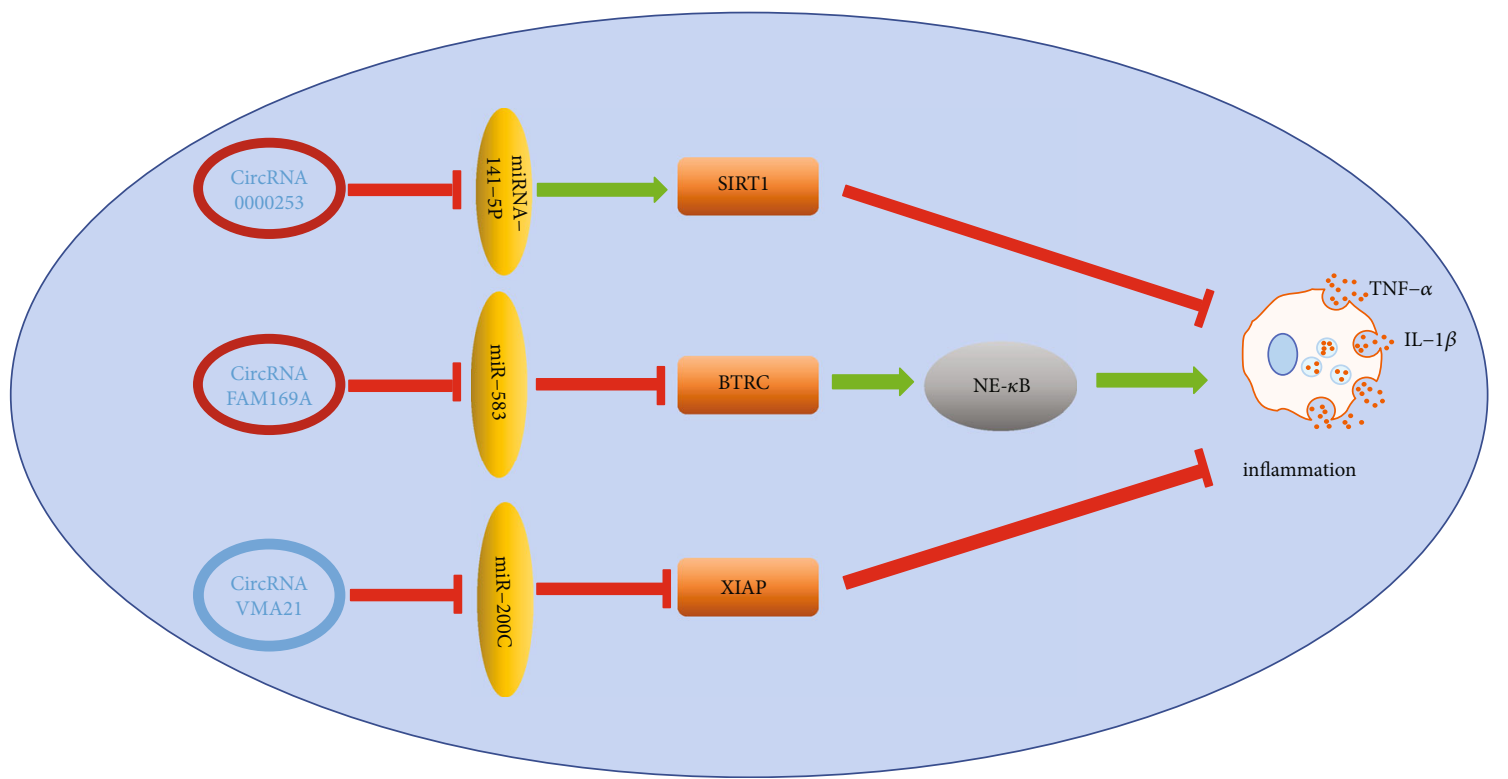

FiguRe 2: Among the reported circRNA, three kinds of circRNA play an important role in intervertebral disc inflammation, among which circRNA0000253 and circRNA FAM169A can promote the synthesis and secretion of intervertebral disc inflammatory cytokines, promoting the occurrence of inflammatory reaction and promoting intervertebral disc degeneration. On the other hand, circRNA VMA21 can inhibit the inflammatory reaction of intervertebral disc and protect the intervertebral disc to a certain extent.

et al. [74] studies have shown that circRNA VMA21 inhibited the synthesis and secretion of inflammatory cytokines by binding miRNA-200C and promoted the expression of X-linked inhibitor of apoptosis protein (XIAP), while XIAP can bind and inhibit the activity of the apoptosis-related protein, particularly caspase3/7/9 [75, 76]. In addition to the role of caspase inhibition, an increasing number of evidence indicated that XIAP can regulate inflammation. Downregulation of XIAP facilitates the proinflammatory effect of TNF- $\alpha$ and excessive IL- $1 \beta$ secretion, causing severe sterile inflammation [77-81]. The etiology of intervertebral disc degeneration is multigenic. However, an increasing body of evidence showed that excessive secretion of inflammatory cytokines is the chief factor in IDD [9, 82] (Figure 2).

4.2. ECM Metabolism. ECM is a noncellular, complex, and highly dynamic structure, regulating cellular function, facilitating communication between diverse cells, and maintaining homeostasis [83]. Among its complex component, aggrecan and collagen are crucial for its integrity and function [84]. However, matrix metalloproteinases (MMPs) and a disintegrin and metalloproteinases with thrombospondin motifs (ADAMTS) are closely related with ECM degradation [61, 81]. Similarly, accumulating studies have shown that some circRNAs are involved in the regulation of ECM metabolism. Up to now, 10 circRNAs are reported that are related to ECM metabolism, of which 5 circRNAs promote ECM catabolism (circRNA_0000253, circRNA TIMP2, circRNA-001653, circRNA-CIDN, and circRNA-104670). On the contrary, there are 4 circRNA (circRNA VMA21, circERCC2, circSEMA4B, and circ-4099) promoted the anabolism of ECM (Figure 3). However, the role of circRNA FAM169A may remain controversial. Inflammatory cytokines can upregulate the production of catabolic factors such as MMPs to facilitate the degradation of ECM [85, 86]. Therefore, Guo et al. [44] revealed that circ-FAM169A regulate NF- $\kappa$ B pathway-induced IL- $1 \beta$ and TNF- $\alpha$ production via the miR-583/BTRC signaling 


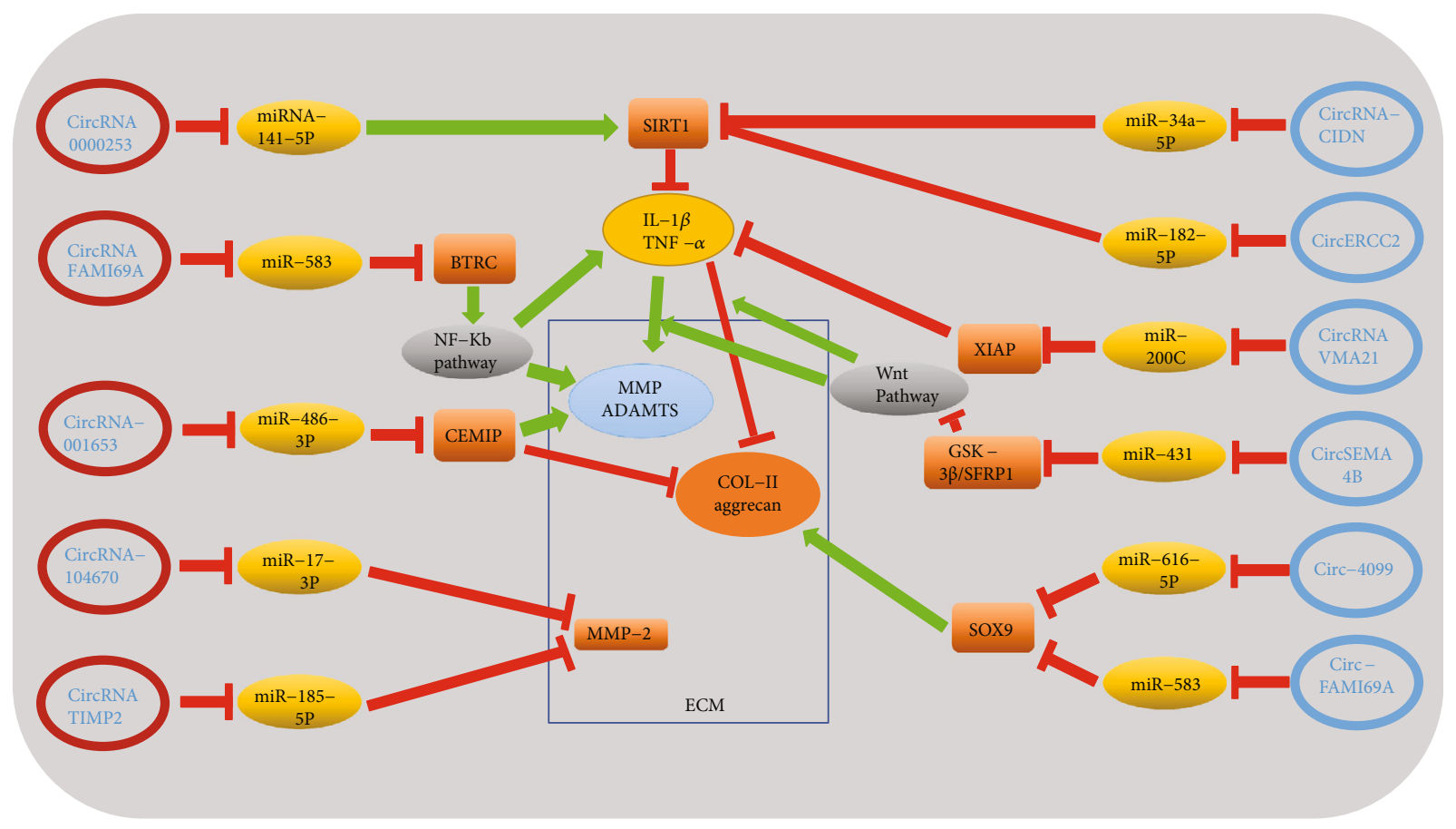

FIGURE 3: Red-labeled circRNA has a negative effect on the metabolism of extracellular matrix and can promote the catabolism of ECM. However, blue-labeled circRNA plays a role in promoting the anabolism of ECM. Song et al. [73] suggested that circRNA_0000253 could promote IDD by adsorbing miRNA-141-5p and downregulating SIRT1. Guo et al. [44] revealed that circ-FAM169A promotes IDD development via the miR-583/BTRC signaling pathway. However, Li et al. [87] proved that circ-FAM169A promote NPC proliferation and extracellular matrix synthesis by the circ-FAM169A-miR-583 pathway. Cui and Zhang [88] suggested that circ_001653 inhibited ECM synthesis of NPCs in IDD by the miR-486-3p/CEMIP axis. Song et al. [89] proved that circRNA_104670 is upregulated in human IDD tissues and upregulates MMP-2 by directly sponging miR-17-3p. Guo et al. [90] revealed that circ-TIMP2 promoted dysmetabolism of ECM via the miR-185-5p-MMP2 pathway. Xiang et al. [91] revealed that circRNA-CIDN downregulated the expression of ECM catabolism enzymes (MMP-3 and MMP-13) and upregulated the level of anabolism markers (collagen II and aggrecan) by the miR-34a5p/SIRT1 pathway to retard compression-induced ECM degradation. Xie et al. [52] demonstrated that circERCC2 can mitigate ECM degradation via targeting miR-182-5p/SIRT1 axis. Cheng et al. [74] revealed that circVMA21 could alleviate inflammatory cytokineinduced dysmetabolism of ECM through the miR-200c-XIAP pathway. Wang et al. [92] demonstrated that circSEMA4B acts as a sponge and a ceRNA form miR431 in NPCs and competes with GSK-3 $\beta$ and SFRP1 for miR-431 binding, thus inhibiting IL- $1 \beta$-induced degenerative process in NPCs through Wnt signaling. Wang et al. [84] revealed that circular RNA circ-4099 regulates ECM synthesis by blocking miR-616-5p inhibition of Sox9.

pathway to upregulate the expression of MMP-13 and ADAMTS-5 and downregulate the expression of collagen II and aggrecan to promote IDD. However, Li et al. [87] proved that circ-FAM169A alleviate IDD development by promoting NPC proliferation and extracellular matrix synthesis via the circ-FAM169A-miR-583 pathway. In their study, they believe that miR-583 can bind to downstream mRNA such as MMP2, insulin-like growth factor 1 (IGF1), and SRY-related high mobility group box 9 (Sox9) possibly to regulate the metabolism of ECM, NPC apoptosis, and proliferation. Overall, the above two different results show that one RNA may play opposite roles in the development of IDD. This result may be due to the different stages of disease development, the inherent dual role of circRNAs, and the limitations in the understanding. Until now, almost all known circRNAs are involved in regulating the metabolism of ECM, indicating that ECM metabolism disorder may be an intermediate process in the pathological mechanism of IDD, and promoting ECM synthesis by circRNA may delay or even reverse the development of IDD, which may be a new breakthrough in the diagnosis and treatment of IDD in the future.

4.3. Apoptosis. Currently, programmed cell death (PCD) and necrosis are the main forms of cell death. Apoptosis, also known as type I PCD, is featured by chromosomal concentration, cell shrinkage, DNA degradation, and apoptotic body formation and relies on caspase [93]. It causes continuous cell loss throughout life and is closely associated with the degenerative diseases [94-96]. Recently, several studies pay attention to the relationship between circRNA and apoptosis of NP cells (Figure 4). For example, Song et al. [73] have shown that circRNA 0000253 was confirmed to facilitate IDD by inhibiting miRNA-141-5p and downregulating SIRT1, thus increasing the expression of apoptosis-related proteins such as caspase3/7/9 to promote apoptosis. Caspases are a protein family that plays a crucial role in regulating cell apoptosis (caspase-3/6/7/8/9 in mammals) and inflammation (caspase-1/4/5/12 in humans and caspase-1/11/12 in mice) [97]. SIRT1 is a highly conserved nicotinamide 


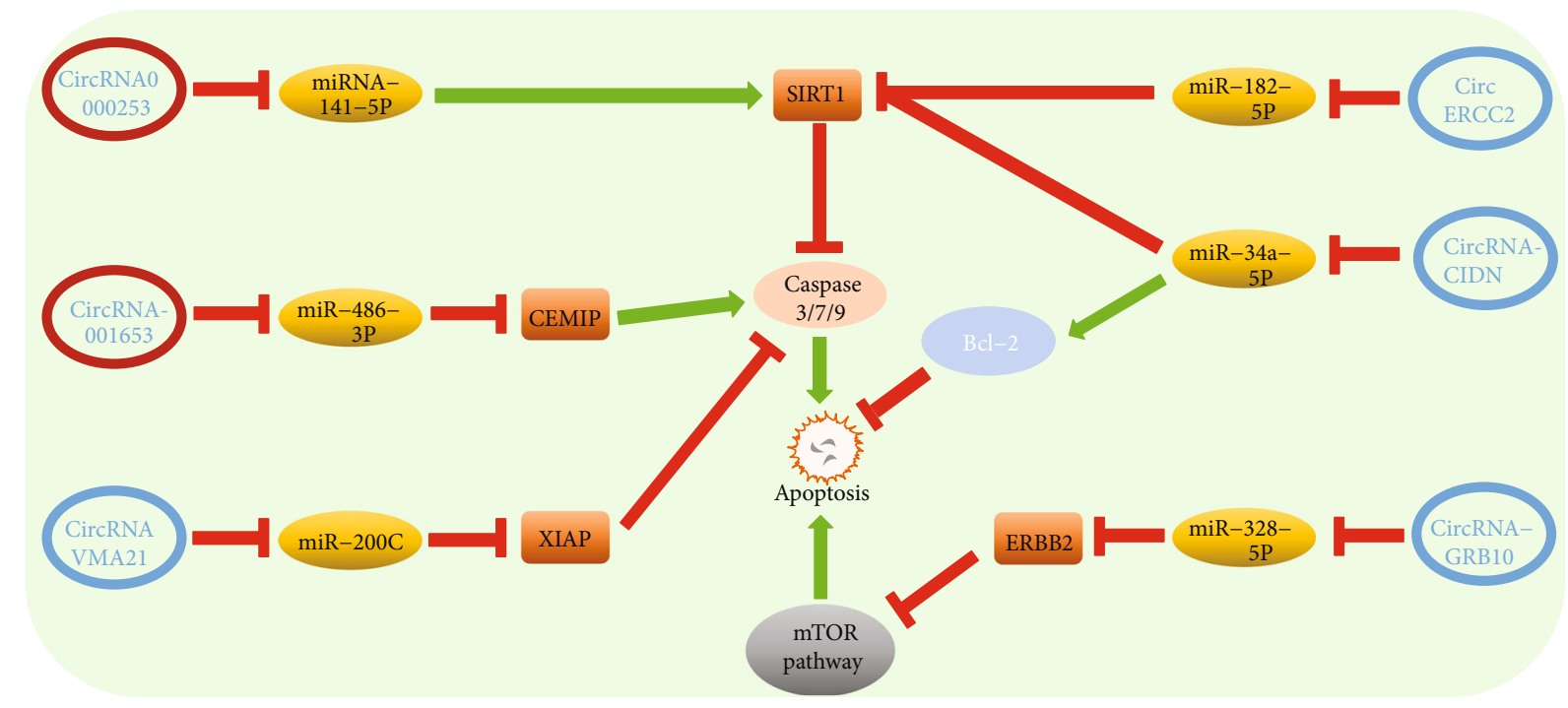

FIGURE 4: (a) CEMIP: cell migration-inducing hyaluronan-binding protein is closely related to cellular invasion, proliferation, and motility [88] (b) XIAP: XIAP belongs to the inhibitor-of-apoptosis proteins (IAP) that represent a family of endogenous caspase inhibitors and can bind and directly inhibit the activity of the three most important apoptosis effector caspases: caspase-3, caspase-7, and caspase-9 [74]. (c) ERBB2: ERBB2 is tyrosine kinase receptor which inhibits autophagy via the formation of a complex with Beclin 1, which is a key regulator of autophagy [82]. (d) SIRT1 is a $\mathrm{NAD}^{+}$-dependent deacetylase that can reduce apoptosis in several different cells [73]. (e) Bcl2 belongs to the antiapoptotic protein [91].

TABLE 1: The role of circRNAs in intervertebral disc degeneration.

\begin{tabular}{lccc}
\hline circRNA & Pathway & Function & Reference \\
\hline circRNA_0000253 & miRNA-141-5p/SIRT1 & Apoptosis/inflammation/ECM catabolism \\
circRNA VMA21 & miR-200c/XIAP & Anti-inflammation/antiapoptosis/ECM anabolism \\
circRNA-GRB10 & miR-328-5p/ERBB2 & Antiapoptosis & [73] \\
circRNA TIMP2 & miR-185-5p/MMP2 & ECM catabolism & [82] \\
circRNA-001653 & miR-486-3p/CEMIP & Apoptosis/ECM catabolism \\
circRNA FAM169A & miR-583/BTRC & Inflammation/ECM catabolism \\
circRNA FAM169A & miR-583/Sox9 & Antiapoptosis/ECM anabolism \\
circRNA-CIDN & miR-34a-5p/SIRT1 & Antiapoptosis/ECM anabolism \\
circERCC2 & miR-182-5p/SIRT1 & Antiapoptosis/ECM anabolism \\
circSEMA4B & miR-431/GSK-3ß/SFRP1 & ECM anabolism \\
circRNA-104670 & miRNA-17-3p/MMP2 & ECM catabolism \\
circ-4099 & miR-616-5p/Sox9 & ECM anabolism \\
\hline
\end{tabular}

adenine dinucleotide- $(\mathrm{NAD}-)^{+}$dependent lysine deacetylase and has been related with longevity and lifespan extension, which widely involved in signaling pathways of inflammation, cell proliferation, and death [98, 99]. Except that the circRNA-miRNA-SIRT1-caspase pathway has been proved regulating apoptosis of NPCs many times [52, 91], Cui and Zhang [88] suggested that circ_001653 could bind miR-486-3p, facilitating the expression of downstream CEMIP and caspase3/7/9, increasing NPC apoptosis. On the contrary, Cheng et al. [74] revealed that circular RNA VMA21 protects against IDD. Circular RNA VMA21 target miR-200c and XIAP to alleviate caspaseinduced NPC apoptosis. Besides, there are also reports that show overexpressed circ-GRB10 inhibit miR-328-5p to upregulate the expression of ERBB2 to alleviate apoptosis of NPCs via the mTOR pathway [82]. Therefore, it seems feasible to alleviate IDD by circRNA to alleviate NPC excessive apoptosis.

\section{Conclusion}

IDD is seriously harmful to human health, but the pathomechanism of IDD is not fully understood. With people's understanding of disease, the influence of gene on disease has become a new research hotspot, and increasingly, scholars believe that genetic factor is one of the most important causes of IDD. circRNAs, as a kind of ncRNAs, can bind to the target miRNAs to regulate gene replication, transcription, and translation. With the deepening of research, there are more evidences indicated that circRNAs 
play an important role in the regulation of inflammation, NPC apoptosis and ECM metabolism, etc. (Table 1). Inflammation plays a critical role in the development of IDD. The overexpression of proinflammatory cytokines and the onset of an inflammatory environment induce the cascade of degenerative events that may eventually cause pain. The current treatment of IDD also mainly focuses on the control and elimination of local inflammatory response to relieve symptoms [100-102]. Therefore, it is very promising that circRNA regulates the levels of inflammatory factors and related proteins to relieve pain and delay the development of IDD. Besides, almost all known circRNAs are involved in regulating the metabolism of ECM. We speculate that ECM metabolism disorder may be an intermediate process in the pathological mechanism of IDD. Consequently, intervening the progress of IDD by regulating the metabolism of ECM at the level of genes seems to be a more promising option. However, we still have a lot of confusion about the circRNAs. For example, in the etiology of IDD, how to determine the key circRNAs? In the development of IDD, does different key circRNAs play a leading role at different stages? What factors affect circRNAs in the process of participating in the development of the disease? Therefore, the relationship between circRNAs and disease still needs to be further explored, and we speculate that circRNAs may become indicators of early diagnosis for IDD and a new target for preventing, delaying, or even reversing the pathological process of IDD at the genetic level in the future.

\section{Consent}

Consent is not applicable.

\section{Conflicts of Interest}

The authors declare that the research was conducted in the absence of any commercial or financial relationships that could be construed as a potential conflict of interest.

\section{Authors' Contributions}

Hao Li, Lijun Tian, and Jianhua Li contributed equally to this work.

\section{Acknowledgments}

Thanks are due to anyone who participated in the review. The present study was supported by grants from the National Natural Science Foundation of China (grant nos. 31670983 and 31900967).

\section{References}

[1] J. Hartvigsen, M. J. Hancock, A. Kongsted, Q. Louw, M. L. Ferreira, and S. Genevay, "What low back pain is and why we need to pay attention," Lancet (London, England)., vol. 391, no. 10137, pp. 2356-2367, 2018.
[2] N. Patrick, E. Emanski, and M. A. Knaub, "Acute and chronic low back pain," The Medical Clinics of North America., vol. 98, no. 4, pp. 777-789, 2014.

[3] N. N. Knezevic, K. D. Candido, J. W. S. Vlaeyen, J. Van Zundert, and S. P. Cohen, "Low back pain," Lancet (London, England)., vol. 398, pp. 78-92, 2021.

[4] GBD 2017 Disease and Injury Incidence and Prevalence Collaborators, "Global, regional, and national incidence, prevalence, and years lived with disability for 354 diseases and injuries for 195 countries and territories, 1990-2017: a systematic analysis for the Global Burden of Disease Study 2017," Lancet (London, England), vol. 392, pp. 1789-1858, 2018.

[5] A. Cieza, K. Causey, K. Kamenov, S. W. Hanson, S. Chatterji, and T. Vos, "Global estimates of the need for rehabilitation based on the Global Burden of Disease Study 2019: a systematic analysis for the Global Burden of Disease Study 2019," Lancet (London, England)., vol. 396, pp. 2006-2017, 2020.

[6] M. V. Risbud and I. M. Shapiro, "Role of cytokines in intervertebral disc degeneration: pain and disc content," Nature Reviews Rheumatology., vol. 10, pp. 44-56, 2014.

[7] K. Luoma, H. Riihimäki, R. Luukkonen, R. Raininko, E. Viikari-Juntura, and A. Lamminen, "Low back pain in relation to lumbar disc degeneration," Spine Phila $\mathrm{Pa}$, vol. 2000, no. 25, pp. 487-492, 2000.

[8] G. Livshits, M. Popham, I. Malkin, P. N. Sambrook, A. J. Macgregor, and T. Spector, "Lumbar disc degeneration and genetic factors are the main risk factors for low back pain in women: the UK Twin Spine Study," Annals of the Rheumatic Diseases., vol. 70, pp. 1740-1745, 2011.

[9] M. Miscusi, C. Carnevali, L. Ricciardi, S. Miglietta, V. Petrozza, and J. Cacciotti, "Histomorphology and immunohistochemical patterns in degenerative disc disease and clinical-radiological correlations: a prospective study," European Spine Journal: official publication of the European Spine Society, the European Spinal Deformity Society, and the European Section of the Cervical Spine Research Society., vol. 29, pp. 1410-1415, 2020.

[10] M. A. Adams, "Biomechanics of back pain," Acupuncture in Medicine: journal of the British Medical Acupuncture Society., vol. 22, pp. 178-188, 2004.

[11] M. C. Battié and T. Videman, "Lumbar disc degeneration: epidemiology and genetics," The Journal of Bone and Joint Surgery American Volume., vol. 88, suppl_2, pp. 3-9, 2006.

[12] M. C. Battié, T. Videman, and E. Parent, "Lumbar disc degeneration: epidemiology and genetic influences," Spine Phila Pa, vol. 29, no. 23, pp. 2679-2690, 1976.

[13] M. C. Battié, T. Videman, J. Kaprio, L. E. Gibbons, K. Gill, and H. Manninen, "The Twin Spine Study: contributions to a changing view of disc degeneration," The Spine Journal: official journal of the North American Spine Society., vol. 9, pp. 47-59, 2009.

[14] J. Fairbank, "Prolapsed intervertebral disc," BMJ Clinical Research Ed, vol. 336, pp. 1317-1318, 2008.

[15] S. Hu, Y. Fu, B. Yan, Z. Shen, and T. Lan, "Analysis of key genes and pathways associated with the pathogenesis of intervertebral disc degeneration," Journal of Orthopaedic Surgery and Research., vol. 15, 2020.

[16] W. Guo, B. Zhang, Y. Li, H. Q. Duan, C. Sun, and Y. Q. Xu, "Gene expression profile identifies potential biomarkers for 
human intervertebral disc degeneration," Molecular Medicine Reports, vol. 16, pp. 8665-8672, 2017.

[17] A. K. Schubert, J. J. Smink, M. Arp, J. Ringe, A. A. Hegewald, and M. Sittinger, "Quality assessment of surgical disc samples discriminates human annulus fibrosus and nucleus pulposus on tissue and molecular level," International Journal of Molecular Sciences, vol. 19, no. 6, p. 1761, 2018.

[18] G. Ni, G. Liu, and K. Yu, "Identification of key genes associated with the effect of osmotic stimuli on intervertebral discs using microarray analysis," Oncology Letters., vol. 14, pp. 4249-4255, 2017.

[19] Y. H. Zhang, J. Song, L. Shen, and J. Shao, "Systematic identification of lncRNAs and circRNAs-associated ceRNA networks in human lumbar disc degeneration," Biotechnic \& Histochemistry, vol. 94, pp. 606-616, 2019.

[20] Z. Li, X. Chen, D. Xu, S. Li, M. T. V. Chan, and W. K. K. Wu, "Circular RNAs in nucleus pulposus cell function and intervertebral disc degeneration," Cell Proliferation., vol. 52, no. 6, article e12704, 2019.

[21] Z. Huo, H. Li, L. Tian et al., "Construction of a Potentially Functional circRNA-miRNA-mRNA Network in Intervertebral Disc Degeneration by Bioinformatics Analysis," BioMed Research International, vol. 2021, Article ID 8352683, 21 pages, 2021.

[22] J. Zang, D. Lu, and A. Xu, "The interaction of circRNAs and RNA binding proteins: an important part of circRNA maintenance and function," Journal of Neuroscience Research, vol. 98, pp. 87-97, 2020.

[23] V. N. Wijayathunga, S. F. Tanner, J. P. Ridgway, and R. K. Wilcox, "An in vitro study of the intervertebral disc structure using $3 \mathrm{~T}$ magnetic resonance imaging," Spine Phila $\mathrm{Pa}$, vol. 44, no. 11, pp. 793-800, 2019.

[24] D. R. Eyre and H. Muir, "Quantitative analysis of types I and II collagens in human intervertebral discs at various ages," Biochimica et Biophysica Acta, vol. 492, pp. 29-42, 1977.

[25] M. K. Chelberg, G. M. Banks, D. F. Geiger, and T. R. Oegema Jr., "Identification of heterogeneous cell populations in normal human intervertebral disc," Journal of Anatomy, vol. 186, Part 1, pp. 43-53, 1995.

[26] P. J. Roughley, "Biology of intervertebral disc aging and Degeneration," Spine Phila Pa, vol. 29, no. 23, pp. 26912699, 2004.

[27] P. Roughley, D. Martens, J. Rantakokko, M. Alini, F. Mwale, and J. Antoniou, "The involvement of aggrecan polymorphism in degeneration of human intervertebral disc and articular cartilage," European Cells \& Materials., vol. 11, pp. 1-7; discussion 7, 2006.

[28] A. R. Poole, "Biologic markers and disc degeneration," The Journal of Bone and Joint Surgery American Volume., vol. 88, suppl_2, pp. 72-75, 2006.

[29] N. Inoue and O. A. Espinoza, "Biomechanics of intervertebral disk degeneration," The Orthopedic Clinics of North America., vol. 42, no. 4, pp. 487-499, 2011.

[30] C. P. L. Paul, K. S. Emanuel, I. Kingma, A. J. van der Veen, R. M. Holewijn, and P. A. Vergroesen, "Changes in intervertebral disk mechanical behavior during early degeneration," Journal of Biomechanical Engineering, vol. 140, 2018.

[31] T. Oichi, Y. Taniguchi, Y. Oshima, S. Tanaka, and T. Saito, "Pathomechanism of intervertebral disc degeneration," JOR Spine., vol. 3, 2020.
[32] N. V. Vo, R. A. Hartman, P. R. Patil, M. V. Risbud, D. Kletsas, and J. C. Iatridis, "Molecular mechanisms of biological aging in intervertebral discs," Journal of Orthopaedic Research: official publication of the Orthopaedic Research Society., vol. 34, pp. 1289-1306, 2016.

[33] F. Marchand and A. M. Ahmed, "Investigation of the laminate structure of lumbar disc anulus fibrosus," Spine Phila $\mathrm{Pa}$, vol. 1990, no. 15, pp. 402-410, 1990.

[34] C. A. Pezowicz, P. A. Robertson, and N. D. BroomIntralamellar relationships within the collagenous architecture of the annulus fibrosus imaged in its fully hydrated state," Journal of Anatomy, vol. 207, pp. 299-312, 2005.

[35] Y. C. Huang, J. P. Urban, and K. D. Luk, "Intervertebral disc regeneration: do nutrients lead the way?," Nature Reviews Rheumatology., vol. 10, pp. 561-566, 2014.

[36] M. P. Grant, L. M. Epure, R. Bokhari, P. Roughley, J. Antoniou, and F. Mwale, "Human cartilaginous endplate degeneration is induced by calcium and the extracellular calcium-sensing receptor in the intervertebral disc," European Cells \& Materials., vol. 32, pp. 137-151, 2016.

[37] H. M. Xu, F. Hu, X. Y. Wang, and S. L. Tong, "Relationship between apoptosis of endplate microvasculature and degeneration of the intervertebral disk," World Neurosurgery, vol. 125, pp. e392-e397, 2019.

[38] J. P. Urban, S. Smith, and J. C. Fairbank, "Nutrition of the intervertebral disc," Spine Phila Pa, vol. 29, pp. 2700-2709, 2004.

[39] T. R. Oegema Jr., "Biochemistry of the intervertebral disc," Clinics in Sports Medicine., vol. 12, pp. 419-439, 1993.

[40] P. Cazzanelli and K. Wuertz-Kozak, "MicroRNAs in intervertebral disc degeneration, apoptosis, inflammation, and mechanobiology," International Journal of Molecular Sciences., vol. 21, 2020.

[41] J. Clouet, C. Vinatier, C. Merceron, M. Pot-Vaucel, O. Hamel, and P. Weiss, "The intervertebral disc: from pathophysiology to tissue engineering," Joint, Bone, Spine, vol. 76, pp. 614-618, 2009.

[42] W. R. Jeck and N. E. Sharpless, "Detecting and characterizing circular RNAs," Nature Biotechnology, vol. 32, pp. 453-461, 2014.

[43] K. V. Morris and J. S. Mattick, "The rise of regulatory RNA," Nature Reviews. Genetics, vol. 15, pp. 423-437, 2014.

[44] W. Guo, K. Mu, B. Zhang, C. Sun, L. Zhao, and Z. Y. Dong, "The circular RNA FAM169A functions as a competitive endogenous RNA and regulates intervertebral disc degeneration by targeting miR-583 and BTRC," Cell Death \& Disease., vol. 11, p. 315, 2020.

[45] M. Matsui and D. R. Corey, "Non-coding RNAs as drug targets," Nature Reviews Drug Discovery., vol. 16, pp. 167-179, 2017.

[46] W. Poller, S. Dimmeler, S. Heymans, T. Zeller, J. Haas, and M. Karakas, "Non-coding RNAs in cardiovascular diseases: diagnostic and therapeutic perspectives," European Heart Journal., vol. 39, pp. 2704-2716, 2018.

[47] J. Wang, S. Zhu, N. Meng, Y. He, R. Lu, and G. R. Yan, "ncRNA-encoded peptides or proteins and cancer," Molecular Therapy: the journal of the American Society of Gene Therapy., vol. 27, pp. 1718-1725, 2019.

[48] M. Esteller, "Non-coding RNAs in human disease," Nature Reviews. Genetics, vol. 12, pp. 861-874, 2011. 
[49] X. Liu, L. Che, Y. K. Xie, Q. J. Hu, C. J. Ma, and Y. J. Pei, "Noncoding RNAs in human intervertebral disc degeneration: an integrated microarray study," Genom Data., vol. 5, pp. 80-81, 2015.

[50] P. H. Lan, Z. H. Liu, Y. J. Pei, Z. G. Wu, Y. Yu, and Y. F. Yang, "Landscape of RNAs in human lumbar disc degeneration," Oncotarget, vol. 7, pp. 63166-63176, 2016.

[51] H. L. Sanger, G. Klotz, D. Riesner, H. J. Gross, and A. K. Kleinschmidt, "Viroids are single-stranded covalently closed circular RNA molecules existing as highly base-paired rodlike structures," Proceedings of the National Academy of Sciences of the United States of America., vol. 73, pp. 38523856, 1976.

[52] L. Xie, W. Huang, Z. Fang, F. Ding, F. Zou, and X. Ma, "circERCC2 ameliorated intervertebral disc degeneration by regulating mitophagy and apoptosis through miR-182-5p/SIRT1 axis," Cell Death \& Disease., vol. 10, p. 751, 2019.

[53] C. Ebermann, T. Schnarr, and S. Müller, "Recent advances in understanding circular RNAs,” F1000Res, vol. 9, 2020.

[54] J. Zhu, X. Zhang, W. Gao, H. Hu, X. Wang, and D. Hao, "IncRNA/circRNA-miRNA-mRNA ceRNA network in lumbar intervertebral disc degeneration," Molecular Medicine Reports, vol. 20, pp. 3160-3174, 2019.

[55] G. Liang, Z. Liu, L. Tan, A. N. Su, W. G. Jiang, and C. Gong, "HIF1 $\alpha$-associated circDENND4C promotes proliferation of breast cancer cells in hypoxic environment," Anticancer Research, vol. 37, pp. 4337-4343, 2017.

[56] Y. Shi, C. F. Sun, W. H. Ge, Y. P. Du, and N. B. Hu, "Circular RNA VMA21 ameliorates sepsis-associated acute kidney injury by regulating miR-9-3p/SMG1/inflammation axis and oxidative stress," Journal of Cellular and Molecular Medicine, vol. 24, pp. 11397-11408, 2020.

[57] C. Y. Yu and H. C. Kuo, "The emerging roles and functions of circular RNAs and their generation," Journal of Biomedical Science, vol. 26, 2019.

[58] Y. Li, Q. Zheng, C. Bao, S. Li, W. Guo, and J. Zhao, "Circular RNA is enriched and stable in exosomes: a promising biomarker for cancer diagnosis," Cell Research, vol. 25, pp. 981-984, 2015.

[59] L. Zuo, L. Zhang, J. Zu, Z. Wang, B. Han, and B. Chen, "Circulating circular RNAs as biomarkers for the diagnosis and prediction of outcomes in acute ischemic stroke," Stroke, vol. 51, pp. 319-323, 2020.

[60] S. Li, W. Hu, F. Deng, S. Chen, P. Zhu, and M. Wang, "Identification of circular RNA hsa_circ_0001599 as a novel biomarker for large-artery atherosclerotic stroke," DNA and Cell Biology., vol. 40, pp. 457-468, 2021.

[61] S. Wang, J. Sun, H. Yang, W. Zou, B. Zheng, and Y. Chen, "Profiling and bioinformatics analysis of differentially expressed circular RNAs in human intervertebral disc degeneration," Acta Biochim Biophys Sin Shanghai, vol. 51, pp. 571-579, 2019.

[62] D. J. Gorth, I. M. Shapiro, and M. V. Risbud, "A new understanding of the role of IL-1 in age-related intervertebral disc degeneration in a murine model," Journal of Bone and Mineral Research: the official journal of the American Society for Bone and Mineral Research., vol. 34, pp. 1531-1542, 2019.

[63] C. Weiler, A. G. Nerlich, B. E. Bachmeier, and N. Boos, "Expression and distribution of tumor necrosis factor alpha in human lumbar intervertebral discs: a study in surgical specimen and autopsy controls," Spine Phila $P a$, vol. 30, pp. 44-53, 2005.

[64] C. L. Le Maitre, J. A. Hoyland, and A. J. Freemont, "Catabolic cytokine expression in degenerate and herniated human intervertebral discs: IL-1beta and TNFalpha expression profile," Arthritis Research \& Therapy., vol. 9, 2007.

[65] J. B. Park, H. Chang, and Y. S. Kim, "The pattern of interleukin-12 and T-helper types 1 and 2 cytokine expression in herniated lumbar disc tissue," Spine Phila $\mathrm{Pa}$, vol. 27, no. 19, pp. 2125-2128, 2002.

[66] C. L. Le Maitre, A. J. Freemont, and J. A. Hoyland, "The role of interleukin-1 in the pathogenesis of human intervertebral disc degeneration," Arthritis Research \& Therapy., vol. 7, pp. R732-R745, 2005.

[67] N. Specchia, A. Pagnotta, A. Toesca, and F. Greco, "Cytokines and growth factors in the protruded intervertebral disc of the lumbar spine," European Spine Journal: official publication of the European Spine Society, the European Spinal Deformity Society, and the European Section of the Cervical Spine Research Society., vol. 11, pp. 145-151, 2002.

[68] H. Takahashi, T. Suguro, Y. Okazima, M. Motegi, Y. Okada, and T. Kakiuchi, "Inflammatory cytokines in the herniated disc of the lumbar spine," Spine Phila $P a$, vol. 21, no. 2, pp. 218-224, 1996.

[69] I. Cosamalón-Gan, T. Cosamalón-Gan, G. Mattos-Piaggio, V. Villar-Suárez, J. García-Cosamalón, and J. A. Vega-Álvarez, "Inflammation in the intervertebral disc herniation," Neurocirugia English Edition, vol. 32, pp. 21-35, 2021.

[70] S. E. Navone, G. Marfia, A. Giannoni, M. Beretta, L. Guarnaccia, and R. Gualtierotti, "Inflammatory mediators and signalling pathways controlling intervertebral disc degeneration," Histology and Histopathology., vol. 32, pp. 523-542, 2017.

[71] S. Wang, C. Liu, Z. Sun, P. Yan, H. Liang, and K. Huang, "IL$1 \beta$ increases asporin expression via the NF- $\kappa \mathrm{B}$ p65 pathway in nucleus pulposus cells during intervertebral disc degeneration," Scientific Reports, vol. 7, 2017.

[72] F. Zhang, X. Zhao, H. Shen, and C. Zhang, "Molecular mechanisms of cell death in intervertebral disc degeneration review," International Journal of Molecular Medicine., vol. 37, pp. 1439-1448, 2016.

[73] J. Song, Z.-H. Chen, C.-J. Zheng et al., "Exosome-transported circRNA_0000253 competitively adsorbs microRNA-141-5p and increases IDD," Molecular Therapy - Nucleic Acids, vol. 21, pp. 1087-1099, 2020

[74] X. Cheng, L. Zhang, K. Zhang, G. Zhang, Y. Hu, and X. Sun, "Circular RNA VMA21 protects against intervertebral disc degeneration through targeting miR-200c and X linked inhibitor-of-apoptosis protein," Annals of the Rheumatic Diseases., vol. 77, pp. 770-779, 2018.

[75] Q. L. Deveraux, R. Takahashi, G. S. Salvesen, and J. C. Reed, "X-linked IAP is a direct inhibitor of cell-death proteases," Nature, vol. 388, pp. 300-304, 1997.

[76] B. P. Eckelman and G. S. Salvesen, "The human antiapoptotic proteins CIAP1 and cIAP2 bind but do not inhibit caspases," The Journal of Biological Chemistry., vol. 281, pp. 3254-3260, 2006.

[77] S. Wicki, U. Gurzeler, W. Wei-Lynn Wong, P. J. Jost, D. Bachmann, and T. Kaufmann, "Loss of XIAP facilitates switch to TNF $\alpha$-induced necroptosis in mouse neutrophils," Cell Death \& Disease., vol. 7, article e2422, 2016. 
[78] W. W. Wong, J. E. Vince, N. Lalaoui, K. E. Lawlor, D. Chau, and A. Bankovacki, "cIAPs and XIAP regulate myelopoiesis through cytokine production in an RIPK1- and RIPK3dependent manner," Blood, vol. 123, pp. 2562-2572, 2014.

[79] K. E. Lawlor, N. Khan, A. Mildenhall, M. Gerlic, B. A. Croker, and A. A. D'Cruz, "RIPK3 promotes cell death and NLRP3 inflammasome activation in the absence of MLKL," Nature Communications, vol. 6, 2015.

[80] J. E. Vince, W. W. Wong, I. Gentle, K. E. Lawlor, R. Allam, and L. O'Reilly, "Inhibitor of apoptosis proteins limit RIP3 kinase-dependent interleukin-1 activation," Immunity, vol. 36, pp. 215-227, 2012.

[81] M. Yabal, N. Müller, H. Adler, N. Knies, C. J. Groß, and R. B. Damgaard, "XIAP restricts TNF- and RIP3-dependent cell death and inflammasome activation," Cell Reports, vol. 7, pp. 1796-1808, 2014.

[82] W. Guo, B. Zhang, K. Mu, S. Q. Feng, Z. Y. Dong, and G. Z. Ning, "Circular RNA GRB10 as a competitive endogenous RNA regulating nucleus pulposus cells death in degenerative intervertebral disk," Cell Death \& Disease., vol. 9, p. 319, 2018.

[83] A. D. Theocharis, S. S. Skandalis, C. Gialeli, and N. K. Karamanos, "Extracellular matrix structure," Advanced Drug Delivery Reviews., vol. 97, pp. 4-27, 2016.

[84] H. Wang, P. He, H. Pan, J. Long, J. Wang, and Z. Li, “Circular RNA circ- 4099 is induced by TNF- $\alpha$ and regulates ECM synthesis by blocking miR-616-5p inhibition of Sox9 in intervertebral disc degeneration," Experimental \& Molecular Medicine., vol. 50, pp. 1-14, 2018.

[85] J. Wang, D. Markova, D. G. Anderson, Z. Zheng, I. M. Shapiro, and M. V. Risbud, "TNF- $\alpha$ and IL- $1 \beta$ promote a disintegrin-like and metalloprotease with thrombospondin type I motif-5-mediated aggrecan degradation through syndecan-4 in intervertebral disc," The Journal of Biological Chemistry., vol. 286, pp. 39738-39749, 2011.

[86] J. Shi, E. Schmitt-Talbot, D. A. DiMattia, and R. G. Dullea, "The differential effects of IL-1 and TNF-alpha on proinflammatory cytokine and matrix metalloproteinase expression in human chondrosarcoma cells," Inflammation Research: official journal of the European Histamine Research Society, vol. 53, pp. 377-389, 2004.

[87] Y. Li, D. Pan, S. Liu et al., "Identification of circ-FAM169A sponges miR-583 involved in the regulation of intervertebral disc degeneration," Journal of Orthopaedic Translation, vol. 26, pp. 121-131, 2021.

[88] S. Cui and L. Zhang, "circ_001653 silencing promotes the proliferation and ECM synthesis of NPCs in IDD by downregulating miR-486-3p-mediated CEMIP," Molecular Therapy - Nucleic Acids, vol. 20, pp. 385-399, 2020.

[89] J. Song, H. L. Wang, K. H. Song, Z. W. Ding, H. L. Wang, and X. S. Ma, "CircularRNA_104670 plays a critical role in intervertebral disc degeneration by functioning as a ceRNA," Experimental \& Molecular Medicine., vol. 50, 2018.

[90] W. Guo, B. Zhang, C. Sun, H. Q. Duan, W. X. Liu, and K. Mu, "Circular RNA derived from TIMP2 functions as a competitive endogenous RNA and regulates intervertebral disc degeneration by targeting miR-185-5p and matrix metalloproteinase 2," International Journal of Molecular Medicine., vol. 46, pp. 621-632, 2020.

[91] Q. Xiang, L. Kang, J. Wang, Z. Liao, Y. Song, and K. Zhao, "circRNA-CIDN mitigated compression loading-induced damage in human nucleus pulposus cells via miR-34a-5p/ SIRT1 axis," eBioMedicine, vol. 53, article 102679, 2020.

[92] X. Wang, B. Wang, M. Zou, J. Li, G. Lü, and Q. Zhang, "circSEMA4B targets miR-431 modulating IL- $1 \beta$-induced degradative changes in nucleus pulposus cells in intervertebral disc degeneration via Wnt pathway," Biochimica et Biophysica Acta - Molecular Basis of Disease, vol. 1864, pp. 3754-3768, 2018.

[93] C. Q. Zhao, L. S. Jiang, and L. Y. Dai, "Programmed cell death in intervertebral disc degeneration," Apoptosis: an international journal on programmed cell death., vol. 11, pp. 20792088, 2006.

[94] H. E. Gruber and E. N. Hanley Jr., "Analysis of aging and degeneration of the human intervertebral disc. Comparison of surgical specimens with normal controls," Spine Phila Pa, vol. 23, no. 7, pp. 751-757, 1998.

[95] D. G. Anderson and C. Tannoury, "Molecular pathogenic factors in symptomatic disc degeneration," The Spine Journal: official journal of the North American Spine Society., vol. 5, pp. 260s-266s, 2005.

[96] F. Ding, Z. W. Shao, and L. M. Xiong, "Cell death in intervertebral disc degeneration," Apoptosis: an international journal on programmed cell death., vol. 18, pp. 777-785, 2013.

[97] D. R. McIlwain, T. Berger, and T. W. Mak, "Caspase functions in cell death and disease," Cold Spring Harbor Perspectives in Biology., vol. 5, article a008656, 2013.

[98] C. Chen, M. Zhou, Y. Ge, and X. Wang, "SIRT1 and aging related signaling pathways," Mechanisms of Ageing and Development., vol. 187, article 111215, 2020.

[99] B. L. Tang, "Sirt1 and the mitochondria," Molecules and Cells, vol. 39, pp. 87-95, 2016.

[100] V. E. Casiano, A. M. Dydyk, and M. Varacallo, "Back pain," in Treasure Island FL: StatPearls Publishing Copyright (c) 2021, StatPearls, Ed., StatPearls Publishing LLC, 2021.

[101] J. J. Wong, P. Côté, D. A. Sutton, K. Randhawa, H. Yu, and S. Varatharajan, "Clinical practice guidelines for the noninvasive management of low back pain: a systematic review by the Ontario Protocol for Traffic Injury Management (OPTIMa) Collaboration," European Journal of Pain (London, England)., vol. 21, pp. 201-216, 2017.

[102] A. N. Khan, H. E. Jacobsen, J. Khan, C. G. Filippi, M. Levine, and R. A. Lehman Jr., "Inflammatory biomarkers of low back pain and disc degeneration: a review," Annals of the New York Academy of Sciences., vol. 1410, pp. 68-84, 2017. 